et al. (11) is an excellent example of scientists pushing past the traditional limits of their discipline to take advantage of biology, chemistry, and nanotechnology and overcome the shortcomings of clinical applications of antiviral peptides. While the authors focused on the use of stabilized peptides and nanoparticle delivery as prophylactic measures, their cell-based work demonstrating robust inhibition of RSV fusion and entry (11) suggests that the peptides could be adapted as antiviral therapeutics for persons with RSV infection. Moreover, the formation of hairpin-linked 6 -HBs is a classic hallmark of many fusogenic enveloped viruses, including Ebola virus, HIV, and SARS coronavirus, which all bring viral and cellular membranes together via formation of a $6-\mathrm{HB}$ in the same fashion as RSV (17). It is possible that the present findings made with RSV could be adapted to combat a host of pathogenic human viruses, both as prophylactic agents and as antiviral treatments.

\section{Acknowledgments}

The authors were supported by grants from the NIH (T32 AI095202 to S.P. Katen; R01 AI076983 to T.S. Dermody).
Address correspondence to: Terence S. Dermody, Elizabeth B. Lamb Center for Pediatric Research, Vanderbilt University School of Medicine, D7235 MCN, 1161 21st Avenue South, Nashville, Tennessee 37232, USA. Phone: 615.343.9943; Fax: 615.343.7659; E-mail: terry.dermody@vanderbilt.edu.

1. World Health Organization. Initiative for Vaccine Research, Acute respiratory infections. WHO Web site. http://apps.who.int/vaccine_research/diseases/ ari/en/index.html. Updated September 1, 2009. Accessed March 10, 2014.

2. Andabaka T, Nickerson JW, Rojas-Reyes MX, Rueda JD, Bacic Vrca V, Barsic B. Monoclonal antibody for reducing the risk of respiratory syncytial virus infection in children. Cochrane Database Syst Rev. 2013;4:CD006602.

3. Hall CB, et al. Respiratory syncytial viral infection in children with compromised immune function. N Engl J Med. 1986;315(2):77-81.

4. Haynes LM. Progress and challenges in RSV prophylaxis and vaccine development. J Infect Dis. 2013; 208(suppl 3):S177-S183.

5. McLellan JS, Yang Y, Graham BS, Kwong PD. Structure of respiratory syncytial virus fusion glycoprotein in the postfusion conformation reveals preservation of neutralizing epitopes. J Virol. 2011; 85(15):7788-7796.

6. McLellan JS, et al. Structure of RSV fusion glycoprotein trimer bound to a prefusion-specific neutralizing antibody. Science. 2013;340(6136):1113-1117.

7. Douglas JL, et al. Inhibition of respiratory syncytial virus fusion by the small molecule VP-14637 via specific interactions with F protein. J Virol. 2003; 77:5054-5064.
8. Razinkov V, Gazumyan A, Nikitenko A, Ellestad G, Krishnamurthy G. RFI-641 inhibits entry of respiratory syncytial virus via interactions with fusion protein. Chem Biol. 2001;8(7):645-659.

9. Garg R, Latimer L, Simko E, Gerdts V, Potter A, van den Hurk S. Induction of mucosal immunity and protection by intranasal immunization with a respiratory syncytial virus subunit vaccine formulation. J Gen Virol. 2014;95(pt 2):301-306.

10. Rigter A, et al. A protective and safe intranasal RSV vaccine based on a recombinant prefusion-like form of the F protein bound to bacterium-like particles. PLoS One. 2013;8(8):e71072.

11. Bird GH, et al. Mucosal delivery of a double-stapled RSV peptide prevents nasopulmonary infection. J Clin Invest. 2014;124(5):2113-2124.

12. Kilby JM, et al. Potent suppression of HIV-1 replication in humans by T-20, a peptide inhibitor of gp41mediated virus entry. Nat Med. 1998;4(11):1302-1307.

13. Lambert DM, et al. Peptides from conserved regions of paramyxovirus fusion $(\mathrm{F})$ proteins are potent inhibitors of viral fusion. Proc Natl Acad Sci US A. 1996;93(5):2186-2191.

14. Schafmeister C, Po J, Verdine GL. An all-hydrocarbon cross-linking system for enhancing the helicity and metabolic stability of peptides. J Am Chem Soc. 2000;122(24):5891-5892.

15. Luo Y, Wang Q. Recent development of chitosanbased polyelectrolyte complexes with natural polysaccharides for drug delivery. Int J Biol Macromol. 2014;64C:353-367.

16. Menon JU, Ravikumar P, Pise A, Gyawali D, Hsia CC, Nguyen KT. Polymeric nanoparticles for pulmonary protein and DNA delivery. Acta Biomaterialia. 2014;pii:S1742-7061(14)00052-X.

17. Melikyan GB. Common principles and intermediates of viral protein-mediated fusion: the HIV-1 paradigm. Retrovirology. 2008;5:111.

\title{
A VISTA on PD-1H
}

\section{Yang Liu}

Centers for Cancer and Immunology Research, Children's Research Institute, Children's National Medical Center, Washington, DC, USA.

\begin{abstract}
Three years ago, two research groups independently identified a previously undescribed $\mathrm{T}$ cell cosignaling molecule; one referred to it as V-domain Ig suppressor of $T$ cell activation (VISTA), and the other used the term programmed death-1 homolog (PD-1H). Recombinant and ectopically expressed PD-1H functions as a coinhibitory ligand for $\mathrm{T}$ cell responses. However, the function of endogenous PD-1H is not clear. In this issue of the JCI, Flies and colleagues demonstrate that endogenous PD-1H on both $T$ cells and APCs serves as a coinhibitory molecule for $T$ cell activation and provide further support for targeting PD-1H as a therapeutic strategy for transplantation and cancers.
\end{abstract}

Life is never dull, especially if you are interested in $\mathrm{T}$ cell cosignaling molecules. The identification of new members of this family can lead not only to new paradigms in immune recognition, but can also affect the treatment of cancer and autoimmune

Conflict of interest: The author has declared that no conflict of interest exists.

Citation for this article: J Clin Invest. 2014; 124(5):1891-1893. doi:10.1172/JCI75798. diseases (1-4). Among the newest additions to the $\mathrm{T}$ cell cosignaling family is an immunoglobulin superfamily member that bears two different names due to its independent discoveries three years ago: $\mathrm{V}$-domain Ig suppressor of $\mathrm{T}$ cell activation (VISTA) and programmed death-1 homolog (PD-1H).

Wang et al. named this molecule VISTA (5), based on significant sequence homology with $\mathrm{B} 7$ homologue 1 (B7H1) and the ability of recombinant VISTA-Fc protein and ectopic expression of VISTA on APCs or tumor cells to inhibit $\mathrm{T}$ cell proliferation. In an independent study, Flies and colleagues identified the same molecule and named it PD-1H due to greater overall sequence and gene structure similarities with programmed death-1 (PD-1) (6).

\section{Same target, different results}

Remarkably, the antibodies produced against the molecule by Wang and colleagues and Flies et al. had starkly different effects. Whereas Wang et al. found that their anti-VISTA antibody exacerbated EAE, Flies et al. found that their antiPD-1H antibody suppressed graft-versushost diseases (GVDH) to such an extent that fully allogeneic bone marrow chimera mice (BALB/c to lethally irradiated C57BL/6 mice) could be obtained following a single dose of anti-PD-1H antibody 
A

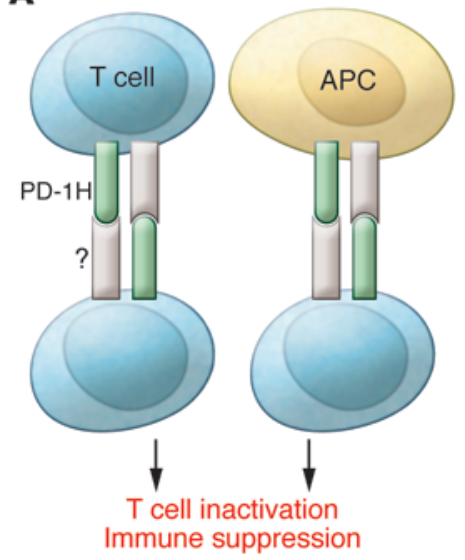

B

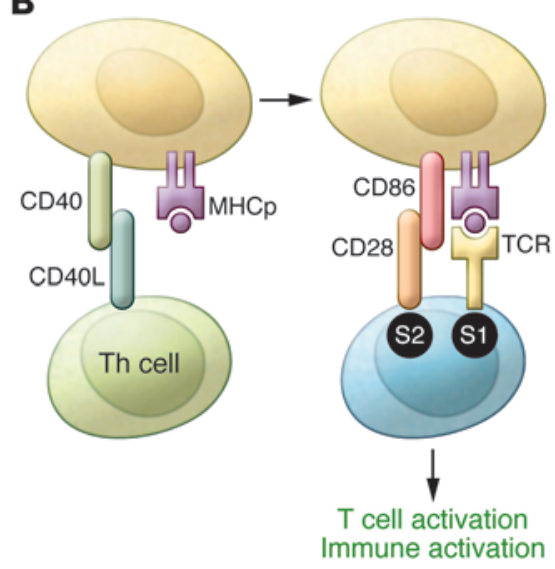

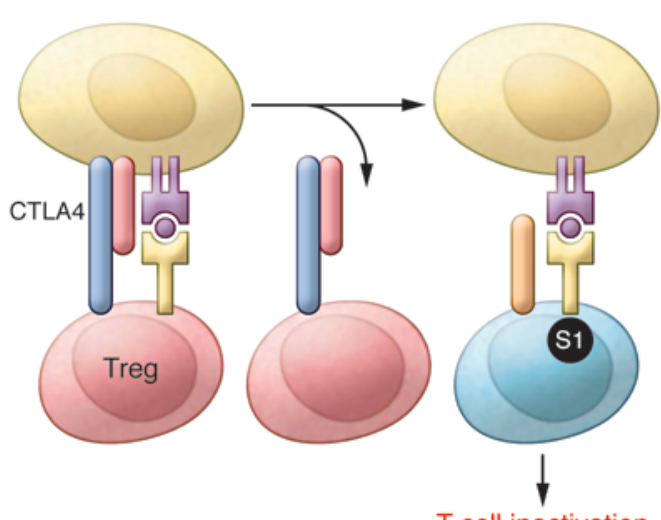

T cell inactivation Immune suppression

\section{Figure 1}

Models of T cell/T cell interaction. (A) A new PD-1H-centric model. PD-1H on the surface of T cells and APCs interacts with an unknown receptor(s) on $T$ cells to directly inactivate $T$ cells and confer immune suppression. (B) Classic models of immune activation and immune suppression through modulation of costimulatory activity of APCs. For immune activation, interaction of CD40L expressed on Th cells with CD40 on APCs induces expression of costimulatory molecules, including CD86 and others. The T cells that recognize the same APCs are activated as they are provided with both signal 1 (S1), the cognate antigens (MHC plus peptide [MHCp]), and signal 2 (S2), the costimulatory signal. During immune suppression, Tregs use CTLA-4 to rip CD28 ligands from the surface of APCs. The T cells that recognize the same APC are suppressed as they are provided with only $\mathrm{S} 1$, which inactivates $\mathrm{T}$ cells through death and anergy $(17,18)$.

at the onset of transplantation. The efficacy of anti-PD-1H in GVDH has not been achieved by other biologics.

Why is there such a difference in the effects of the anti-PD-1H and anti-VISTA antibodies, given that they target the same molecule? One of the first clues to reconcile the antibody-specific differences was the demonstration by Wang et al. that their anti-VISTA antibody shows almost undetectable levels of VISTA after T cell activation, while Flies et al. observed substantial elevation of PD-1H on both $\mathrm{CD}^{+}$ and $\mathrm{CD}^{+} \mathrm{T}$ cells after activation. These fickle antibodies highlight what students of immunology have learned from mice: it is not a good idea to discern biology of a molecule by its antibodies (7-10). In this issue, Flies et al. (11) elucidate the biological function of $\mathrm{PD}-1 \mathrm{H}$ using mice with targeted mutation of the gene encoding PD-1H.

\section{PD-1H on both T cells and APC inhibits $T$ cell function}

Flies et al. (11) obtained PD-1H-deficient mice from a regional resource center (Mutant Mouse Regional Resource Centers [MMRRC], UCD, Davis, California, USA). Despite broad expression of $P d 1 b$ in multiple lineages of hematopoietic cells, Flies and colleagues found no difference in the composition of any lineage of PD-1H-expressing cells, ruling out a major role for PD-1H in hematopoiesis and $\mathrm{T}$ cell development. Compared with their WT littermates, $P d 1 b^{-/-}$mice exhibited an increased frequency of activated $\mathrm{T}$ cells as they aged. This result is consistent with an overall $\mathrm{T}$ cell inhibitory function of PD-1H. Furthermore, $P d 1 b^{-/-}$mice had enhanced susceptibility to concanvalin A-induced hepatitis, but had greater resistance to ectopically transplanted glioma, especially after radiotherapy.

Using purified $\mathrm{T}$ cells from WT and $P d 1 b^{-/-}$mice, Flies and colleagues clearly demonstrated that $\mathrm{T}$ cells lacking PD-1H are more responsive to cognate antigen and surrogate $\mathrm{T}$ cell receptor ligands. In addition, the enhanced activation of T cells by $P d 1 b^{-/-}$APCs suggests that PD-1H on APCs inhibits T cells regardless of their $P d 1 b$ genotype. Therefore, $\mathrm{PD}-1 \mathrm{H}$ is a coinhibitory molecule regardless of its cellular distribution. Together, these results pave the way for immune modulation by anti-PD-1H antibodies for autoimmune diseases and transplantation as described in this issue (11) and previously by Flies et al. (6).

\section{Remaining questions}

The data reported to date on PD- $1 \mathrm{H}$ have raised intriguing issues in relation to its fundamental mechanism of immune recognition, receptor, and function of unique intracellular domain.

First, in relation to the issue of immune recognition, Flies et al. mixed $\mathrm{T}$ cells and APCs from WT and $P d 1 b^{-/-}$mice and showed that PD-1H on both $\mathrm{T}$ cells and
APCs can suppress T cell activation (11). Because $T$ cells both express and respond negatively to $\mathrm{PD}-1 \mathrm{H}$, it is easy to envision a suppressive $\mathrm{T}$ cell/ $\mathrm{T}$ cell interaction directly through PD-1H (Figure 1A). This potentially direct $\mathrm{T}$ cell/T cell interaction is very different from the known mechanisms of $T$ cell crosstalk, including those that mediate both immunological help and immune suppression, which are based solely on modification of APCs (Figure 1B). Th cells help induce costimulatory activity on APCs $(12,13)$. Conversely, Tregs suppress T cell activation by ripping off costimulatory molecules from APCs (14). A general mechanism for direct $\mathrm{T}$ cell/T cell suppression was previously invoked (15), but proof of such an interaction has eluded immunologists. With the identification of $\mathrm{PD}-1 \mathrm{H}$ as a coinhibitor that is abundantly expressed on activated $\mathrm{T}$ cells, it is not hard to envision that PD-1H on neighboring $\mathrm{T}$ cells enables them to tune each other's response to cognate antigen (Figure 1A). Such a tuning mechanism can also be extended interactions between $\mathrm{PD}-1 \mathrm{H}$-expressing $\mathrm{T}$ cells and APCs (Figure 1A).

The second unresolved issue is the nature of the $\mathrm{PD}-1 \mathrm{H}$ receptor. The elevated response of $P d 1 b^{-/-} \mathrm{T}$ cells to $\mathrm{PD}-1 \mathrm{H}$ as reported by Flies and colleagues, along with the data from Wang et al. demonstrating that recombinant VISTA-Fc robustly inhibits $\mathrm{T}$ cell activation, suggests that both $\mathrm{T}$ cells and APCs have a PD-1H/VISTA recep- 
tor or receptors. A direct binding partner of $\mathrm{PD}-1 \mathrm{H}$ has yet to be shown, and a putative receptor remains to be identified.

Third, notwithstanding the expression of PD-1H on T cells, most data to date can be accounted for by evoking PD-1H strictly as an inhibitory ligand, as previously suggested by Ceeraz et al. (16). This interpretation, while advantageous for its simplicity, fails to account for the existence of the large and very unique intracellular domain of the molecule. Wang et al. noted that VISTA is highly conserved in organisms ranging from fungi to humans (5); however, while the extracellular IgV domain of VISTA shows homology with $\mathrm{B} 7 \mathrm{H} 1$, no relative of the large intracellular domain has been found, suggesting that this intracellular domain may have evolved independently of other cosignaling molecules. The unusual evolutionary conservation of the intracellular domain of $\mathrm{PD}-1 \mathrm{H}$ hints at a functional importance for this part of the molecule, but its uniqueness shields any clue as to what that function may be. With these outstanding issues and the strong effect of targeting PD- $1 \mathrm{H}$ in transplantation, autoimmune diseases, and cancer, it is safe to assume that we have not heard the last from this protein.

\section{Acknowledgments}

I thank Pan Zheng and Dashuang Shi for helpful discussion. This work is supported by grants from the NIH (CA58033 and AI64350).

Address correspondence to: Yang Liu, Centers for Cancer and Immunology Research, Children's Research Institute, Children's National Medical Center, 111 Michigan Avenue NW, Washington, DC 20010, USA. Phone: 202.476.5849; Fax: 202.476.6498; E-mail: yaliu@cnmc.org.

1. Genovese MC, et al. Abatacept for rheumatoid arthritis refractory to tumor necrosis factor $\alpha$ inhibition. N Engl J Med. 2005;353(11):1114-1123.

2. Hwu P. Treating cancer by targeting the immune system. N Engl J Med. 2010;363(8):779-781.

3. Kremer JM, et al. Treatment of rheumatoid arthritis by selective inhibition of T-cell activation with fusion protein CTLA4Ig. N Engl J Med. 2003 ; 349(20):1907-1915

4. Ribas A. Tumor immunotherapy directed at PD-1. NEngl J Med. 2012;366(26):2517-2519.

5 . Wang L, et al. VISTA, a novel mouse Ig superfamily ligand that negatively regulates $\mathrm{T}$ cell responses. J Exp Med. 2011;208(3):577-592.

6. Flies DB, Wang S, Xu H, Chen L. Cutting edge: A monoclonal antibody specific for the programmed death-1 homolog prevents graft-versus-host disease in mouse models. J Immunol. 2011;187(4):1537-1541.

7. Bachmann MF, Gallimore A, Jones E, Ecabert B, Acha-Orbea H, Kopf M. Normal pathogen-specific immune responses mounted by CTLA-4-deficient T cells: a paradigm reconsidered. Eur J Immunol. 2001; 31(2):450-458.

8. Bachmann MF, Kohler G, Ecabert B, Mak TW, Kopf M. Cutting edge: lymphoproliferative disease in the absence of CTLA- 4 is not T cell autonomous. JImmunol. 1999;163(3):1128-1131.

9. Liu Y. Is CTLA-4 a negative regulator for T-cell activation? Immunol Today. 1997;18(12):569-572.

10. Nguyen TV, Ke Y, Zhang EE, Feng GS. Conditional deletion of Shp2 tyrosine phosphatase in thymocytes suppresses both pre-TCR and TCR signals. Jimmunol. 2006;177(9):5990-5996.

11. Flies DB, et al. Coinhibitory receptor $\mathrm{PD}-1 \mathrm{H}$ preferentially suppresses $\mathrm{CD}^{+} \mathrm{T}$ cell-mediated immunity. J Clin Invest. 2014;124(5):1966-1975.

12. Guerder S, Matzinger P. A fail-safe mechanism for maintaining self-tolerance. J Exp Med. 1992; 176(2):553-564

13. Ridge JP, Di Rosa F, Matzinger P. A conditioned dendritic cell can be a temporal bridge between a $\mathrm{CD}^{+} \mathrm{T}$ - helper and a T-killer cell [see comments]. Nature. 1998;393(6684):474-478.

14 . Wing K, et al. CTLA-4 control over Foxp $3^{+}$regulatory T cell function. Science. 2008;322(5899):271-275.

15. Janeway CA. Autoimmune disease: immunotherapy by peptides? Nature. 1989;341(6242):482-483.

16. Ceeraz S, Nowak EC, Noelle RJ. B7 family checkpoint regulators in immune regulation and disease. Trends Immunol. 2013;34(11):556-563.

17. Liu Y, Janeway CA, Janeway CA Jr. Interferon $\gamma$ plays a critical role in induced cell death of effector T cell: a possible third mechanism of self-tolerance. J Exp Med. 1990;172(6):1735-1739.

18. Schwartz RH. Costimulation of T lymphocytes: the role of CD28, CTLA-4, and B7/BB1 in interleukin-2 production and immunotherapy. Cell. 1992; 71(7):1065-1068

\title{
Cryptococcosis: a model for the understanding of infectious diseases
}

\author{
John R. Perfect \\ Department of Medicine, Division of Infectious Diseases, Duke University Medical Center, Durham, North Carolina, USA.
}

\begin{abstract}
The increase in immunosuppressed patient populations has correlated with a rise in clinical fungal infections, including cryptococcosis. Patient outcome following Cryptococcus infection is linked to initial fungal burden in cerebrospinal fluid (CSF) and fungal clearance following treatment; however, the role of the pathogen in disease prognosis is poorly defined. In this issue of the JCI, Sabiiti and colleagues have directly correlated phenotypic traits of Cryptococcus neoformans with clinical outcome of infected patients. A better understanding of both the host and pathogen contributions to disease etiology will provide more options for targeted treatment strategies.
\end{abstract}

\section{Clinical impact}

Cryptococcosis, which results from infection by Cryptococcus neoformans and Cryptococcus gattii, exploded onto the clinical

Conflict of interest: John R. Perfect has served as a consultant for or been on the advisory committee of Astellas, Pfizer, F2G, Viamet, and Scynexis.

Citation for this article: $J$ Clin Invest. 2014; 124(5):1893-1895. doi:10.1172/JCI75241. scene in three major outbreaks. In 1978, Kauffman and Blumer marked the first outbreak as the "awakening giant of mycology" (1). The frequency of cryptococcosis rose in concert with an enlarging immunosuppressed population as the result of advanced medical therapies for serious underlying diseases. The rapid increase in cryptococcosis was the "canary in the coal mine" sentinel finding for the fragility of immunosuppressed patients and our lack of precision in manipulating the immune system. The second major outbreak occurred in the mid-1980s as the immunosuppressed HIV pandemic gripped the world, with the number of patients affected and the effects of the virus on immunity reflected in the appearance of an incredible number of cryptococcal cases, estimated at 1 million per year worldwide (2). The third major outbreak of cryptococcosis occurred around 2000 in the Pacific Northwest as the result of C. gattii infections. This outbreak has challenged our understanding of new recombinant (hypervirulent) strains (3) and of the potential influences of climate on changing fungal ecology. Cryptococcus has become a "new-age" pathogen that reaches every level of clini- 